\title{
Cooperative and Reliable ARQ Protocols for Energy Harvesting Wireless Sensor Nodes
}

\author{
Marco Tacca, Member, IEEE, Paolo Monti, Member, IEEE, and Andrea Fumagalli, Member, IEEE
}

\begin{abstract}
One class of wireless sensor networks makes use of sensor nodes that recharge their batteries by harvesting energy from the surrounding environment. Being continuously recharged, the battery does not need to be replaced regularly and the sensor node is maintenance-free. A key module in such sensor network solutions is the data link automatic repeat request (ARQ) protocol, which must be designed to reliably deliver sensor nodes data at the minimum energy cost. With this objective in mind, two ARQ protocol classes are compared. In one class, each sensor node operates individually. In the other, the concept of cooperative communications is adopted, whereby neighboring sensor nodes help each other during the retransmission process.

It is shown that the use of cooperative $A R Q$ protocols in energy harvesting sensor networks enables sensor nodes to balance their energy consumption to match their own battery recharge rate. In turn, a balanced energy consumption-to-recharge rate ratio has the potential to improve the network throughput. Both classes of ARQ protocols are analyzed and compared. Estimated throughput gains are discussed under various network scenarios.
\end{abstract}

Index Terms-Sensor networks, energy harvesting, radio cooperation, ARQ protocol, greedy algorithm.

\section{INTRODUCTION}

$\mathbf{N}$ ETWORKS of wireless sensor nodes are often used to monitor distributed parameters in the environment. These parameters are related to a variety of applications, such as security, patient monitoring, chemical and biological hazard detection [1]. The benefits of using wireless sensor networks include reduced installation costs, ability to rapidly reconfigure the data acquisition procedure, and safe deployment in hostile environments [2]-[7]. Networked sensor nodes can jointly perform large sensing tasks, thus greatly improving the accuracy and scope of the information provided to the user.

Some solutions rely on sensor nodes that are powered up by limited life-time batteries, which are periodically replaced to provide long-term sensor node operation [1]. Others envision sensor nodes harvesting their energy from the surrounding environment (e.g., solar energy, vibrations, acoustic noise, microwave power). These maintenance-free solutions - examples are the PicoRadio project at Berkeley, the $\mu$ AMPS

Manuscript received November 1, 2005; revised October 30, 2006, and January 17, 2007; accepted January 24, 2007. The associate editor coordinating the review of this paper and approving it for publication was W. Liao. This paper was presented in part at the Second European Workshop on Sensor Networks (EWSN '05), Istanbul, Turkey, January-February 2005. This work was supported in part by NSF Grants No. ECS-0225528, CNS-0435429 and CNS-0435393.

The authors are with the Open Networking Advanced Research (OpNeAR) Laboratory, Erik Jonsson School of Engineering and Computer Science, The University of Texas at Dallas, Richardson, TX 75080, USA (e-mail: \{mtacca, paolo, andreaf\}@utdallas.edu).

Digital Object Identifier 10.1109/TWC.2007.05878. project (with base-station) at MIT, the WSSN project at ICT Vienna, and the GAP4S project at UT Dallas - aim at low cost sensor nodes densely deployed across the area of interest. The foreseen power dissipation level at the sensor node is in the order of $100 \mu \mathrm{W}$. At these power levels it may be possible to energy-scavenge or harvest directly from the environment [8]-[10].

The objective in this paper is to determine the maximum throughput generated by energy harvesting sensor nodes inside the footprint of a base-station. The base-station - which may be mobile - gathers data from the sensor nodes, constitutes the access point to a wider communication network, and is assumed not to have stringent power constraints. More precisely, the objective is to determine the maximum saturation throughput from the sensor nodes to the base-station while offering a reliable and fair delivery of the sensed information. Saturation throughput is defined as the maximum value of the offered load that can be sustained by the sensor nodes without exceeding their energy harvesting rate [11]. The term reliable is used to indicate that a data link automatic repeat request (ARQ) protocol is used to cope with transmission errors. The term fair is used to indicate that all the sensor nodes must be able to deliver an amount of data that is proportional to a reference value.

Two classes of ARQ protocols are considered. The first is the conventional class of ARQ protocols, whereby the source (sensor node) retransmits its own data frames until they are successfully delivered to the base-station. The second class takes advantage of cooperative radio communications [12][18]. In this second class of ARQ protocols one or more relays (i.e., selected sensor nodes) assist the source during the retransmission process. In simple terms, the relay instead of the source - is requested to retransmit the data frame to the base-station when the earlier transmission attempt made by the source is not successful. With this retransmission mechanism, it is as if sensor nodes could borrow energy from one another and balance their energy consumption to match their own battery recharge rate. In turn, a balanced energy consumption-to-recharge rate ratio has the potential to improve the network throughput. A second advantage of using cooperative ARQ protocols derives from their inherent retransmission mechanism. The relay offers its spatial diversity and, if it is located between the source and the base-station, data frames are (re)transmitted over a shorter range, thus reducing the required amount of transmitted energy.

For the cooperative ARQ protocol class, relays must be chosen for each source to best balance energy usage at the sensor nodes. This problem is formulated in its most general 
form - i.e. allowing multiple relays to be assigned to the same source - using linear programming (LP). A special sub-case is obtained by constraining each source to make use of one relay only, i.e., the one-relay $A R Q$ protocol class. For the onerelay sub-case, a sub-optimal greedy solution is presented. The solution is based on a sorting function applied to choosing the relay for each source.

The saturation throughput of both cooperative and noncooperative ARQ protocols is discussed for a number of (GAP4S) network scenarios, e.g., varying the sensor node transmission power, base-station footprint size, path loss exponent, and radio frequency used. It is confirmed that introducing cooperation in the ARQ protocols enables the sensor node to transmit data more efficiently by using lower energy levels. Under constrained energy budget at the sensor node, higher saturation throughput can thus be achieved when compared to non-cooperative ARQ protocols. The one-relay ARQ protocols class is found to be a good trade-off between network throughput and protocol complexity.

\section{Two Classes of ARQ Protocols}

Consider a network of wireless sensor nodes located inside the footprint of the base-station. Taking advantage of the base-station's presence, the following medium access control solution is used. Using the downlink (base-station to sensor node) control channel, the base-station schedules when each sensor node must either transmit or receive. By following the base-station's provided schedule, collision-free channel access is achieved. Any scheduling solution that guarantees a fair transmission (as defined in Section I) on the uplink may be used. To keep the study general, no further assumption is made on the medium access control scheduler. Transmission errors may be frequent on the uplink (sensor node to base-station) channel as the sensor node power budget limits the achievable SNR value. To overcome these transmission errors two classes of ARQ protocols are considered. The two classes of ARQ protocols are defined next.

\section{A. The Class of $A R Q-N C$ Protocols}

The ARQ-NC is the class of (conventional) non-cooperative protocols. Upon a request from the base-station, the sensor node transmits its data frame directly to the base-station. Data frames are encoded to provide error detection and, optionally, correction capabilities at the base-station. The base-station replies with a positive acknowledgment (ACK) frame only upon the successful reception of a data frame. A timer is used at the sensor node to trigger retransmission if the ACK frame is not received timely. The sensor node retransmits the data frame until it is received correctly at the base-station and a positive ACK frame is received at the sensor node.

\section{B. The Class of ARQ-C Protocols}

The ARQ-C is the class of cooperative protocols that take advantage of the broadcast nature of the uplink channel to reach the base-station via spatial diversity. Fig. 1 depicts how the ARQ-C protocols work. Notice that, in general, sensor nodes may act as sources (when transmitting their own data

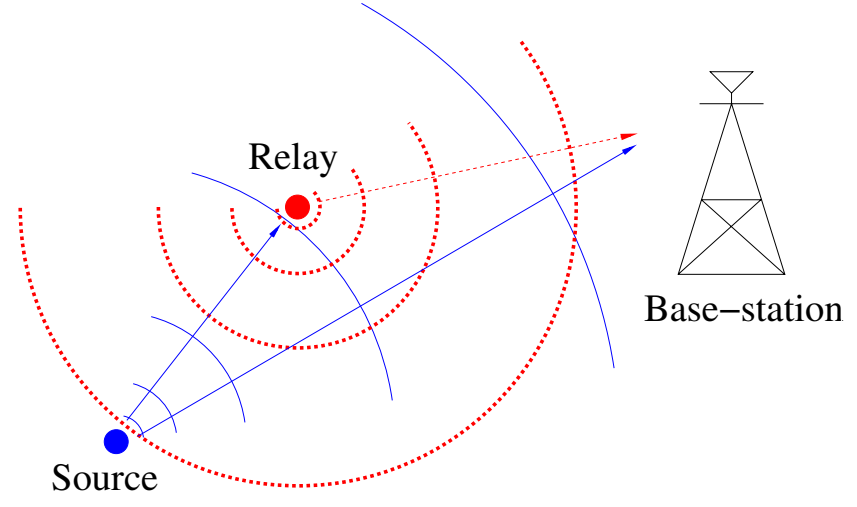

Fig. 1. Cooperation between two sensor nodes.

frames) and as relays (when transmitting data frames generated by other sensor nodes). When scheduling the transmission and reception functions of the sensor nodes, the base-station must indicate which sensor node acts as the source and which acts as the relay. Assume for a moment that the relay successfully overhears the data frame transmission performed by the source and stores a copy of the data frame temporarily. Both source and relay make use of a timer. Both timers are set at the end of the data frame transmission performed by the source. The timer at the relay is set to expire first. If the data frame transmission from the source to the base-station is successful, the base-station responds with an ACK frame, the relay discards the temporary data frame copy, and a new transmission cycle begins. Upon the unsuccessful reception of a data frame transmitted by the source the base-station does not send the ACK frame. The timer at the relay expires first, triggering the retransmission of the data frame copy at the relay. If the data frame transmission from the relay to the base-station is successful the ACK frame is sent and a new transmission cycle begins. Of course, the relay may help only when it correctly overhears the data frame transmitted by the source. If the base-station does not hear from the relay, it is safe to assume that the transmission of the data frame from the source to the relay was not successful. It is also possible that the relay retransmission attempt to the base-station was unsuccessful. Under either circumstances the ACK frame is not sent, and the transmission cycle for the same data frame must start all over again, triggered by the timer expiration at the source.

As already mentioned, a careful choice of the relay may increase the probability of successfully delivering the data frame to the base-station without requiring additional retransmission attempts. In addition, the relay offers its own energy to help with the source's data frame transmission. For improved load and energy consumption balancing, multiple sensor nodes may be chosen to act as relay for the same source. Likewise, the same sensor node may act as relay for multiple sources. When multiple relays are assigned to the same source, it is assumed that only one of them will be "active" during one transmission cycle. This choice eliminates unnecessary reception energy consumption at the other relays. In practice, when scheduling, the base-station selects the active relay for each data frame transmission proportionally to some predefined distribution 
values. Note that the required intelligence to perform this choice is entirely residing at the base-station. Sensor nodes are simply ordered by the base-station when they must transmit and overhear.

It must be noted that the ARQ-C protocol solution just described has some interesting similarity with multi-hop solutions. However, these two solutions should not be confused. In fact, the latter is a layer 3 solution, which requires routing protocols at the sensor nodes and ACK frame exchange at each hop to ensure correct information delivery. The former is a layer 2 solution, whereby the base-station determines which sensor node makes the next (re)transmission attempt and does not require the relay to send any ACK frame to the source.

\section{Assessing Saturation Throughrut}

The two classes of ARQ protocols are compared using saturation throughput as the metric of interest. As already mentioned, the saturation throughput is defined as the maximum offered load - or data frame generation rate - that can be sustained from the sensor nodes to the base-station under two constraints:

- at each sensor node the average energy consumption does not exceed the energy recharge rate,

- data frames are generated at each sensor node proportionally to a given reference value, and must be successfully delivered to the base-station.

It is assumed that other system factors, e.g., wireless channel and electronics bandwidth, buffer capacity at the base-station and sensor nodes, network latency, QoS, etc., do not limit the network throughput. It is also assumed that the energy consumption required to schedule the transmission and reception functions at the sensor nodes is negligible ${ }^{1}$. It is assumed that ACK frames transmitted by the base-station are always successfully received by the source and by the relay. This assumption is reasonable as the SNR of the downlink channel may be considerably higher than the SNR on the uplink channel due to the unconstrained amount of power available at the base-station. The sensor node battery is ideal, i.e., linear recharge rate and unbounded storage capacity. The sensor nodes and the base-station are stationary.

The problem of maximizing the saturation throughput can be formulated using a flow model [19], i.e., keeping track of the number of data frame transmissions at each sensor node. The flow model relies on the following input parameters and variables.

\section{Input:}

- $\mathcal{N}$ : the set of sensor nodes,

- $N=|\mathcal{N}|:$ the number of sensor nodes,

- $P_{i}^{r e c}$ : amount of time average recharge power harvested by sensor node $i$,

- $E_{b_{i}}$ : energy per bit radiated by sensor node $i$,

- $E_{b}^{(R x)}$ : energy consumed by the receiver while receiving and processing one bit,

- $L$ : number of bits per data frame,

- $L_{A C K}$ : number of bits per ACK frame,

${ }^{1}$ If this assumption is not satisfied by the used scheduler the derived equations must be modified accordingly.

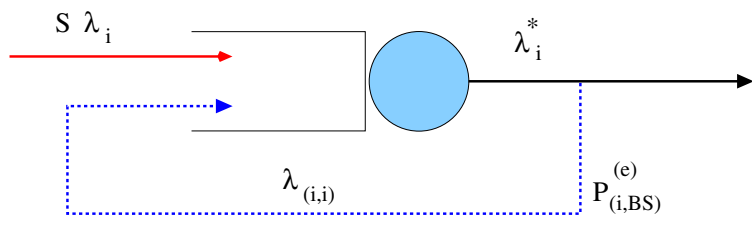

Fig. 2. ARQ-NC: flow model.

- $\gamma$ : the transmission energy overhead at the sensor node, defined as the ratio between the total energy consumption and the radiated energy $E_{b_{i}}$,

- $P_{(i, j)}^{(e)}$ : probability of unsuccessful reception at sensor node $j$ of a data frame sent by sensor node $i$,

- $P_{(i, B S)}^{(e)}$ : probability of unsuccessful reception at the basestation of a data frame sent by sensor node $i$,

- $\lambda_{i}$ : offered load at sensor node $i$ relative to a reference value measured in packets/s.

\section{Variables:}

- $S$ : network saturation throughput,

- $S \cdot \lambda_{i}$ : offered $\operatorname{load}^{2}$ at sensor node $i$,

- $\lambda_{(i, j)}$ : rate of data frames transmitted at source $i$ while having relay $j$ active, measured in packets/s,

- $\bar{\lambda}_{(i, j)}$ : rate of data frames retransmitted by relay $j$ on behalf of source $i$, measured in packets/s,

- $\lambda_{i}^{*}$ : data frame transmission rate at sensor node $i$ (counting both transmission and retransmission attempts) measured in packets/s.

The problem of maximizing the saturation throughput is equivalent to finding the maximum value of $S$ so that, at each sensor node $i$, the offered load $S \cdot \lambda_{i}$ is sustainable without exceeding the sensor node energy recharge rate $P_{i}^{r e c}$. The LP problem formulation for both classes of ARQ protocols is given next.

\section{A. ARQ-NC Protocols}

Fig. 2 shows the flow model of the ARQ-NC protocols. The figure represents the transmission queue at sensor node $i$. With probability $P_{(i, B S)}^{(e)}$ the data frame transmission is not successful, in which case the data frame remains in the transmission queue of sensor node $i$ for the next transmission attempt. The problem of maximizing the saturation throughput $S$ can be formulated as follows [20]:

$$
\max : S
$$

subject to:

$$
\begin{aligned}
\left(\lambda_{i}^{*} \cdot L \cdot E_{b_{i}} \cdot \gamma\right) & +\left(\lambda_{i} \cdot S\right. \\
& \left.\cdot L_{A C K} \cdot E_{b}^{(R x)}\right) \leq P_{i}^{r e c}, \quad \forall i \in \mathcal{N} \\
\lambda_{i}^{*}= & \frac{S \cdot \lambda_{i}}{1-P_{(i, B S)}^{(e)}}, \quad \forall i \in \mathcal{N} .
\end{aligned}
$$

Constraint (1) ensures that the recharge power at each sensor node is sufficient to both (re)transmit the total number of data frames and listen to the ACK frames (one per each successfully delivered data frame) from the base-station.

\footnotetext{
${ }^{2}$ This definition of the offered load is used to model the traffic intensity at every sensor node using a single variable $S$.
} 


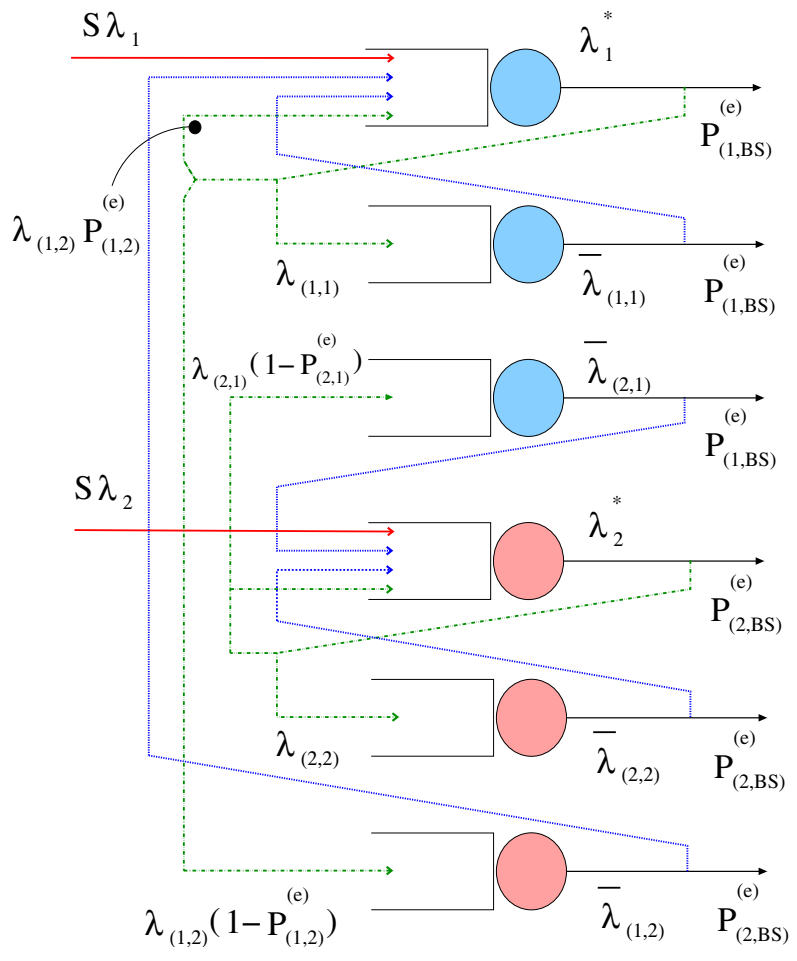

Fig. 3. ARQ-C: flow model.

\section{B. ARQ-C Protocols}

Fig. 3 shows the flow model of the ARQ-C protocols. For simplicity only two sensor nodes are shown. $N+1$ queues are used at each sensor node. Sensor node $i$ has one transmission queue for its own data frames, and a dedicated queue for the data frame copies received from source $j \in \mathcal{N}$. Note that $j=i$ when source $i$ does not have a relay. Having separate queues in the figure simplifies the description of the flow model, but is not strictly necessary in the protocol or sensor node implementation.

Upon the occurrence of an unsuccessful transmission at source $i$, flow $\lambda_{i}^{*}$ is split to reach multiple relays. Let $p_{(i, j)}=$ $\frac{\lambda(i, j)}{\lambda_{i}^{*} P_{(i, B S)}^{(e)}}$ define the probability that the base-station selects relay $j$ when source $i$ begins a transmission cycle. Recall that sensor node $j$ can act as relay only when the transmission from sensor node $i$ to sensor node $j$ is successful. Therefore, $\bar{\lambda}_{(i, j)}=\left(1-P_{(i, j)}^{(e)}\right) \cdot \lambda_{(i, j)}$. The data frame transmission rate at sensor node $i$ is then, $\lambda_{i}^{*}+\sum_{j} \bar{\lambda}_{(j, i)}$.

The amount of power dissipated at relay $j$ to overhear data frames transmitted by source $i$ is proportional to $p_{(i, j)} \cdot \lambda_{i}^{*}=$ $\frac{\lambda_{(i, j)}}{P_{(i, B S)}^{(e)}}$. The total amount of power dissipated at relay $j$ to overhear data frames transmitted by all sources is proportional to $\sum_{i, i \neq j} \frac{\lambda_{(i, j)}}{P_{(i, B S)}^{(e)}}$. Note that the amount of power dissipated while overhearing is irrespective of the successful reception at the relay.

When acting as a source, the amount of power dissipated by sensor node $i$ to receive an ACK frame (one per each successfully delivered data frame) from the base-station is $\lambda_{i} \cdot S \cdot L_{A C K} \cdot E_{b}^{(R x)}$. The amount of power dissipated by sensor node $i$ to receive an ACK frame while acting as the relay for another sensor node, e.g., $j$, is proportional to the sum of two factors. The first factor, $\bar{\lambda}_{(j, i)} \cdot\left(1-P_{(i, B S)}^{(e)}\right)$, is the power dissipated to receive an ACK frame for each data frame successfully retransmitted on behalf of sensor node $j$. The second factor, $\lambda_{(j, i)} \cdot \frac{1-P_{(j), B S)}^{(e)}}{P_{(j, B S)}^{(e)}}$, is the power dissipated to receive an ACK frame for each data frame successfully transmitted by sensor node $j$ to the base-station while sensor node $i$ is acting as its relay. Notice that, as already mentioned in Section II-B, the reception of the ACK frame resets the timer at the relay avoiding unnecessary data frame retransmissions. The problem of maximizing the throughput can be formulated as follows [20]:

\section{$\max : S$}

subject to:

$$
\begin{gathered}
\left(\sum_{j, j \neq i} \frac{\lambda_{(j, i)}}{P_{(j, B S)}^{(e)}}\right) \cdot L \cdot E_{b}^{(R x)}+\left(\lambda_{i}^{*}+\sum_{j} \bar{\lambda}_{(j, i)}\right) \cdot L \\
\cdot E_{b_{i}} \cdot \gamma+\left[\lambda_{i} \cdot S+\sum_{j, j \neq i}\left(\bar{\lambda}_{(j, i)} \cdot\left(1-P_{(i, B S)}^{(e)}\right)+\lambda_{(j, i)}\right.\right. \\
\left.\left.\cdot \frac{1-P_{(j, B S)}^{(e)}}{P_{(j, B S)}^{(e)}}\right)\right] \cdot L_{A C K} \cdot E_{b}^{(R x)} \leq P_{i}^{r e c}, \quad \forall i \in \mathcal{N}
\end{gathered}
$$

$$
\begin{aligned}
& \lambda_{i}^{*}=S \cdot \lambda_{i}+\sum_{j}\left(P_{(i, j)}^{(e)} \cdot \lambda_{(i, j)}\right) \\
&+\sum_{j}\left(P_{(j, B S)}^{(e)} \cdot \bar{\lambda}_{(i, j)}\right), \quad \forall i \in \mathcal{N} \\
& \bar{\lambda}_{(i, j)}=\left(1-P_{(i, j)}^{(e)}\right) \cdot \lambda_{(i, j)}, \quad \forall i, j \in \mathcal{N} \\
& \sum_{j} \lambda_{(i, j)}=P_{(i, B S)}^{(e)} \cdot \lambda_{i}^{*}, \quad \forall i \in \mathcal{N} .
\end{aligned}
$$

Constraint (3) balances the energy harvested at sensor node $i$ with the sum of: $(a)$ the total energy required to both overhear and transmit data frames, and $(b)$ the total energy required to receive ACK frames. Constraint (4) represents the transmission rate of sensor node $i$ own data frames. This is the sum of three terms: new data frames, data frames that the basestation designated to be retransmitted by relay $j$ but were not successfully received at relay $j$, and data frames that source $i$ has to retransmit because the retransmission attempt made by relay $j$ was not successful. Expressions in (5) and (6) are flow conservation constraints. The expression in (6) ensures that $\lambda_{(i, j)} \leq P_{(i, B S)}^{(e)} \cdot \lambda_{i}^{*}$, i.e., $p_{(i, j)} \in[0,1]$.

\section{The Class of One-Relay ARQ Protocols}

The formulation of the maximum saturation throughput for the cooperative ARQ protocols (Section III-B) makes the assumption that different transmission cycles from the same source might use a different sensor node as relay. It may be advantageous to simplify the cooperative ARQ protocols by limiting the number of relays per source to one. The LP problem in Section III-B can be modified to be an integer 


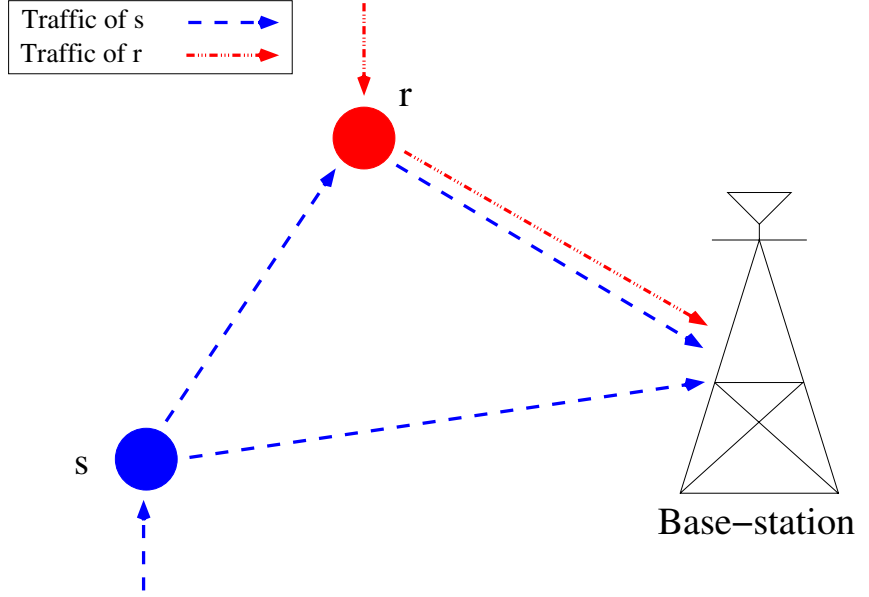

Fig. 4. Three nodes network scenario: sensor node $s$, sensor node $r$ and the base-station $B S$.

(ILP) problem that takes into account this new constraint by adding:

$$
\begin{gathered}
\sum_{j} p_{(i, j)}=1, \quad \forall i \in \mathcal{N} \\
\lambda_{(i, j)} \leq M \cdot p_{(i, j)}, \quad \forall i, j \in \mathcal{N} \\
p_{(i, j)} \in\{0,1\}, \quad \forall i, j \in \mathcal{N}
\end{gathered}
$$

where $M$ is a constant that takes a large value. Constraint (8) is used to force $p_{(i, j)}=1$ whenever $\lambda_{(i, j)} \neq 0$, [20]. To circumvent the computationally intensive ILP solution, an approximate greedy solution is proposed. First, a function is introduced to sort the set of potential relay candidates. Then, the greedy algorithm is described.

\section{A. Sorting Function}

A criterion must be found to identify the relay that is most suitable to cooperate with any given source. The simplified network scenario shown in Fig. 4 is used to construct the sorting function. As already mentioned in Section II-B, sensor nodes may act sometimes as sources and sometime as relays. The network consists of one base-station $B S$ and two sensor nodes $s$ and $r$. The following assumptions are made:

1) both $s$ and $r$ generate data frames to be transmitted to $B S$,

2) when $r$ transmits its own data frames to $B S$, the transmission is always successful,

3) when $s$ transmits its own data frames, the transmission may be unsuccessful,

4) upon an unsuccessful data frame transmission from $s$ to $B S, r$ is always the relay chosen to retransmit the data frame, i.e., $p_{(s, s)}=0, p_{(s, r)}=1$,

5) when $r$ acts as a relay for $s$, its transmission to $B S$ may be unsuccessful,

6) $s$ does not act as relay for $r$, i.e., $p_{(r, s)}=0$,

7) the cost of listening to ACK frames is negligible, i.e., $L_{A C K}=0$ in constraints (1) and (3).

The objective is to define a function that takes as input the coordinates of $s, r, B S$, and the value of $E_{b_{i}}$, and returns an upper bound $\Psi_{(s, r)}$ on the saturation throughput $S$, subject to energy budget constraints. Two cases are considered.

Unlimited energy budget at $\boldsymbol{r}$. In this case the limiting factor is the energy budget at $s$. Considering that $P_{r}^{r e c}=\infty$ and using assumptions 6) and 7), $\lambda_{s}^{*}$ can then be derived from (3) as:

$$
\lambda_{s}^{*}=\frac{P_{s}^{r e c}}{L \cdot E_{b_{s}} \cdot \gamma} .
$$

Using assumption 4), the upper bound $\Psi_{(s, r)}^{(s)}$ on the saturation throughput $S_{(s, r)}^{(s)}$ can be derived from (4)-(6) and (10) as:

$$
\begin{array}{r}
S_{(s, r)}^{(s)} \leq \Psi_{(s, r)}^{(s)}=\frac{\lambda_{s}^{*}}{\lambda_{s}} \cdot\left[1-P_{(s, r)}^{(e)} \cdot P_{(s, B S)}^{(e)}-P_{(r, B S)}^{(e)}\right. \\
\left.\cdot\left(1-P_{(s, r)}^{(e)}\right) \cdot P_{(s, B S)}^{(e)}\right]
\end{array}
$$

Equation (11) takes into account the three energy consumption factors at $s$ that are proportional to the following transmission flows: $(i)$ the flow of data frames transmitted to $B S$, i.e., $\lambda_{s}^{*}$, (ii) the flow of data frames not successfully received at $B S$ nor at $r$, i.e., $\lambda_{s}^{*} \cdot P_{(s, r)}^{(e)} \cdot P_{(s, B S)}^{(e)}$, and $(i i i)$ the flow of data frames that $s$ must retransmit because the retransmission performed by $r$ was not successful, i.e., $\lambda_{s}^{*} \cdot P_{(r, B S)}^{(e)} \cdot\left(1-P_{(s, r)}^{(e)}\right)$. $P_{(s, B S)}^{(e)}$.

Unlimited energy budget at $s$. In this case the limiting factor is the energy budget at $r$. Using assumption 4) and considering that $s$ can sustain any value of throughput $\left(P_{s}^{r e c}=\infty\right), \lambda_{s}^{*}$ can be derived from (4)-(6) as:

$$
\lambda_{s}^{*}=\Psi_{(s, r)}^{(r)} \cdot \lambda_{s} \cdot \alpha^{-1}
$$

where $\Psi_{(s, r)}^{(r)}$ is the upper bound on the saturation throughput $S_{(s, r)}^{(r)}$ and

$$
\alpha=1-P_{s, r}^{(e)} \cdot P_{(s, B S)}^{(e)}-P_{(r, B S)}^{(e)} \cdot\left(1-P_{(s, r)}^{(e)}\right) \cdot P_{(s, B S)}^{(e)} .
$$

Using assumptions 2), 4) and 7), and combining (3) and (12) the upper bound $\Psi_{(s, r)}^{(r)}$ on the saturation throughput $S_{(s, r)}^{(r)}$ can be expressed as follows:

$$
\begin{gathered}
S_{(s, r)}^{(r)} \leq \Psi_{(s, r)}^{(r)}=P_{r}^{r e c} \cdot\left\{\lambda_{s} \cdot \alpha^{-1} \cdot L \cdot E_{b}^{(R x)}+\left[\lambda_{r}+\lambda_{s}\right.\right. \\
\left.\left.\cdot \alpha^{-1} \cdot P_{(s, B S)}^{(e)} \cdot\left(1-P_{(s, r)}^{(e)}\right)\right] \cdot L \cdot E_{b_{r}} \cdot \gamma\right\}^{-1} \cdot(14)
\end{gathered}
$$

Equation (14) takes into account three energy consumption factors at $r$. They are proportional to: $(i)$ the energy spent to receive the flow of data frames from $s$ while taking part in the cooperating process, i.e., $\lambda_{s} \cdot \alpha^{-1} \cdot L \cdot E_{b}^{(R x)}$, (ii) the energy spent to transmit its own data frames to $B S$, i.e., $\lambda_{r} \cdot L \cdot E_{b_{r}} \cdot \gamma$, and (iii) the energy spent to transmit the copies of the data frames successfully received from $s$, i.e., $\lambda_{s} \cdot \alpha^{-1} \cdot P_{(s, B S)}^{(e)}$. $\left(1-P_{(s, r)}^{(e)}\right) \cdot L \cdot E_{b_{r}} \cdot \gamma$

Since the limiting factor in evaluating the saturation throughput is the energy budget available at both $s$ and $r$, the saturation throughput $S$ is upper bounded by the expression:

$$
S \leq \Psi_{(s, r)}=\min \left\{\Psi_{(s, r)}^{(s)}, \Psi_{(s, r)}^{(r)}\right\} .
$$


TABLE I

\section{PSEUdo CODE OF THE GREEDY ALgORITHM}

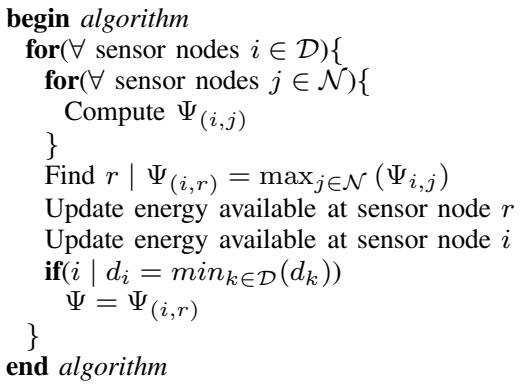

Should $s$ use an ARQ-NC protocol to transmit data frames to $B S$, the upper bound of the saturation throughput $S_{A R Q-N C}$ can be derived from (1), (2) and (10) as follows:

$$
\begin{aligned}
S_{A R Q-N C} \leq \Psi_{(s, s)} & =\frac{\lambda_{s}^{*}\left(1-P_{(s, B S)}^{(e)}\right)}{\lambda_{s}} \\
& =\frac{P_{s}^{r e c}}{L \cdot E_{b_{s}} \cdot \gamma} \cdot \frac{\left(1-P_{(s, B S)}^{(e)}\right)}{\lambda_{s}}
\end{aligned}
$$

where $\lambda_{s}^{*}$ is the total rate of data frames transmitted by $s$ to $B S$ as indicated in (10).

\section{B. The Greedy Algorithm}

The function in (15) takes into account the retransmission attempts made for one sensor node only, and ignores the other sensor nodes' retransmission attempts. For example, it does not capture the practical case where one sensor node may act as the relay for two or more sources. This section details a greedy algorithm that is designed to assign one relay to each source. The assignment algorithm attempts to maximize the saturation throughput while making sure that the energy budget at the sensor node is met. Equation (15) is used to sort the relay candidates for every given source.

The algorithm works as follows. Each sensor node $i \in \mathcal{N}$ is assigned a parameter $d_{i}=\frac{P_{i}^{r e c}}{\lambda_{i} E_{b_{i}} L E\left[t x_{i}\right]} . E\left[t x_{i}\right]$ is the average number of transmission attempts per data frame if $i$ makes use of an ARQ-NC protocol. $E\left[t x_{i}\right]=\frac{\lambda_{i}^{*}}{S \cdot \lambda_{i}}=\frac{1}{1-P_{i, B S}^{(e)}}$ can be derived using (1) and (2). Let $\mathcal{D}$ be the set of sensor nodes ordered by non decreasing values of $d_{i}$. The algorithm considers one sensor node at a time following the order in $\mathcal{D}$. For each $i \in \mathcal{D}$ the algorithm computes $\Psi_{(i, j)}$ when $j \in \mathcal{N}$ is the relay for $i$. The value of $\Psi_{(i, j)}$ is computed from $(15)^{3}$. Sensor node $r$ such that $\Psi_{(i, r)}=\max _{j \in \mathcal{N}}\left(\Psi_{(i, j)}\right)$ is chosen to be the relay for $i$.

The algorithm must consider that a sensor node $r$ may be chosen to be the relay for two (or more) sources. This affects the power budget available at $r$, as explained next. Every time $r$ is chosen to be a relay, its remaining power budget is updated

\footnotetext{
${ }^{3}$ When $i=j, \Psi_{(i, i)}$ is derived using (16).
}

as follows:

$$
\begin{aligned}
P_{r}^{r e c}{ }^{(n e w)} & =P_{r}^{r e c^{(o l d)}}-\Psi_{(i, r)} \cdot \lambda_{i} \cdot L \cdot \alpha^{-1} \\
& \cdot\left[P_{(i, B S)}^{(e)} \cdot\left(1-P_{(i, r)}^{(e)}\right) \cdot E_{b_{r}} \cdot \gamma+E_{b}^{(R x)}\right]
\end{aligned}
$$

where $\alpha$ is given in (13). In addition, every sensor node $i$ must reserve sufficient power budget to transmitting its own data frames. At each step, the algorithm updates the remaining power budget at $i$ using (11) as follows:

$$
\begin{aligned}
& P_{i}^{r e c^{(n e w)}}=P_{i}^{r e c^{(o l d)}}-\Psi_{(i, r)} \cdot \lambda_{i} \cdot\left\{1-P_{(i, r)}^{(e)} \cdot P_{(i, B S)}^{(e)}\right. \\
& \left.\quad-P_{(r, B S)}^{(e)} \cdot\left(1-P_{(i, r)}^{(e)}\right) \cdot P_{(i, B S)}^{(e)}\right\}^{-1} \cdot L \cdot E_{b_{i}} \cdot \gamma
\end{aligned}
$$

where $\alpha$ is given in (13). The algorithm stops after $N$ steps, when one relay $\forall i \in \mathcal{D}$ is chosen. The complexity of the algorithm is $O\left(|\mathcal{N}|^{2}\right)$.

Table I provides a pseudo code description of the algorithm. The description also indicates how to compute an upper bound $(\Psi)$ for the one-relay ARQ protocols saturation throughput. The computation of the upper bound is based on the observation that sensor node $i$ with the smallest value of $d_{i}$ receives the least amount of recharge power and/or has the lowest probability of successfully transmitting data frames to the base-station. The upper bound is a direct consequence of the inequality (15), $S \leq \Psi=\Psi_{(i, r)}$.

\section{Performance Evaluation}

This section reports some saturation throughput results obtained for the two classes of ARQ protocols. The saturation throughput is used to determine under what conditions the ARQ-C protocols outperforms the ARQ-NC protocols and quantify the potential gain. Additionally the two classes of ARQ protocols are compared against a simple energy aware multi-hop protocol. The study is carried out for the GAP4S architecture. A short description of the GAP4S system and the energy aware multi-hop protocol are given first.

\section{A. GAP4S System Description}

This section contains a brief description of the GAP4S architecture. Additional details can be found in [10]. In the GAP4S architecture (Fig. 5) the location of the sensor nodes is restricted to a predetermined footprint surrounding a powerrich base-station. The sensor node sends its generated data directly to the base-station via a wireless uplink channel. Each sensor node recharges its battery using the received microwave (MW) power that is continuously radiated by the base-station in an omnidirectional way ${ }^{4}$. The radiated recharge power is constrained to safety levels. A simple modulation - e.g., On/Off Keying (OOK) - of the MW signal provides the downlink channel from the base-station to the sensor nodes. Among other functions, the downlink control channel is used to distribute slot synchronization, poll the sensor nodes for collision-free uplink transmission, send ACK for the received

\footnotetext{
${ }^{4}$ Although this feature is not explored in this work, the base-station may use directional antennas to ensure best power provisioning and full-duplex connectivity with the sensor nodes.
} 


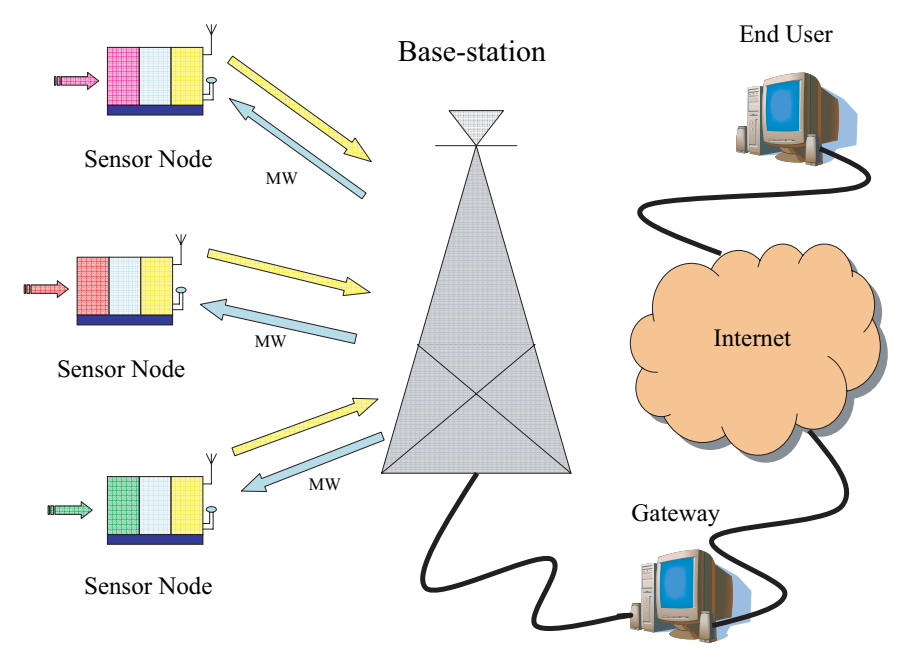

Fig. 5. GAP4S system architecture.

sensor data frames, download software updates, and remotely program sensor nodes for achieving the desired sensing operations. Unlike other solutions, the GAP4S peculiar downlink transmission is not costly to the sensor node in terms of energy as it takes place over the MW recharging channel ${ }^{5}$. Another GAP4S peculiarity is the use of dumb and low powerconsumption sensor nodes, which are entirely driven by the intelligent base-station.

Among other things, the base-station is responsible for ensuring that data frames are collected reliably and fairly from across the entire set of sensor nodes, despite their location within the footprint. Note that both the energy recharge rate at the sensor node and the uplink channel signal-to-noise ratio (SNR) may be affected significantly by the location of the sensor node with respect to the base-station.

\section{B. Energy Aware Multi-Hop Protocol}

The following simple energy aware multi-hop protocol is used as a comparison benchmark for the considered classes of ARQ protocols. The multi-hop protocol is referred to as minimum energy path (MEP) protocol. The multi-hop path from each source to the base-station is computed as follows. For each $(i, j)$ pair, where $i$ is a sensor node and $j$ may be either another sensor node or the base-station, the following energy metric is computed:

$$
\begin{aligned}
e_{(i, j)}= & \frac{1}{1-P_{(i, j)}^{(e)}} \cdot L \cdot \gamma \cdot \frac{E_{b_{i}}}{P_{i}^{r e c}}+L_{A C K} \cdot \frac{E_{b}^{(R x)}}{P_{i}^{r e c}} \\
& +L_{A C K} \cdot \gamma \cdot \frac{E_{b_{j}}}{P_{j}^{r e c}}+\frac{1}{1-P_{(i, j)}^{(e)}} \cdot L \cdot \frac{E_{b}^{(R x)}}{P_{j}^{r e c}} .
\end{aligned}
$$

Equation (19) takes into account four energy consumption factors while transmitting from $i$ to $j:(a)$ the energy consumed at $i$ to successfully transmit a data frame to $j$, i.e., $\frac{1}{1-P_{(i, j)}^{(e)}}$. $L \cdot \gamma \cdot \frac{E_{b_{i}}}{P_{i}^{r e c}},(b)$ the energy consumed at $i$ to receive an ACK

\footnotetext{
${ }^{5}$ This feature corresponds to having $L_{A C K}=0$ in constraints (1) and (3)
}

frame from $j$, i.e., $L_{A C K} \cdot \frac{E_{b}^{(R x)}}{P_{i}^{r e c}},(c)$ the energy consumed at $j$ to transmit an $\mathrm{ACK}$ frame to $i$, i.e., $L_{A C K} \cdot \gamma \cdot \frac{E_{b_{j}}}{P_{j}^{r e c}}$, and $(d)$ the energy consumed at $j$ to successfully receive a data frame from $i$, i.e., $\frac{1}{1-P_{(i, j)}^{(e)}} \cdot L \cdot E_{b}^{(R x)}$. Notice that factor $(b)$ is set to zero when $j=B S$, due to the nature of the GAP4S architecture, i.e., ACK frames are sent using the downlink MW channel that is used to recharge the sensor nodes. Additionally, since the base-station is characterized by a non-stringent power constraint, terms $(c)$ and $(d)$ are set equal to zero when $j=B S$. Each factor in (19) is normalized to the recharging power received at the sensor node. The multihop sequence from each source to the base-station is then computed running a shortest path algorithm [19] based on the metric defined in (19).

\section{Results}

When comparing the two classes of ARQ and multi-hop protocols, the following assumptions are made. Unless otherwise specified, both the uplink and MW downlink frequency is $2.4 \mathrm{GHz}$. Path loss and fading are taken into account in the uplink transmission. Only path loss is taken into account in the MW downlink recharging signal. Unless otherwise specified a path loss coefficient of $n=3$ is used. Fading is assumed to be Rayleigh slow and flat, i.e., the fading coefficients are considered constant over a single frame transmission. The fading experienced by each frame transmission is statistically independent of the fading experienced by any other frame transmission. More details on the models used for path loss and fading can be found in [10].

It is assumed that the MW downlink channel is error free. On the wireless uplink channel, data frames are augmented with a cyclic redundancy code (CRC). Each block contains $B$ bits (including the $\mathrm{CRC}$ bits). The probability of receiving a frame incorrectly (error probability) is a function of both the instantaneous SNR and the CRC. The CRC is used to detect the case of an erroneous codeword decoding, in which case retransmission is required. We assume that the $\mathrm{CRC}$ is able to detect all erroneous codewords. Data frames have fixed length and carry $B=128$ bits of combined data and CRC, which are encoded into $L=256$ bit codewords using a rate-compatible punctured convolutional code (RCPC) with rate $1 / 2$, parent code rate of $1 / 4$, puncturing period of 8 , and memory of 4 [21]. The overall frame error probability $P^{(e)}$ versus SNR at the detector stage of the receiver, $S N R_{\text {rec }}$, is shown in Fig. 6, based on [14]. $S N R_{r e c}$ accounts for both $E_{b_{r}} / N_{0}$ and the receiver noise figure $F$. It is assumed that $F=5 \mathrm{~dB}$. It is assumed that binary PSK with soft decoding is employed.

The values for the transmitting and receiving antenna gain at the base-station and at every sensor node are assumed to be $G_{T}=G_{R}=1$. The recharge power constantly radiated at the base-station is $P_{B S}=10 \mathrm{~W}$. It is assumed that at the sensor node the energy received from the MW channel by the antenna is fully transferred into its battery, and circuitry losses are negligible.

The effects of various energy consumption factors at the sensor node, i.e., analog-digital conversion, processing, power management and coding are all taken into account and combined using parameter $\gamma$ introduced in Section III. Consistently 


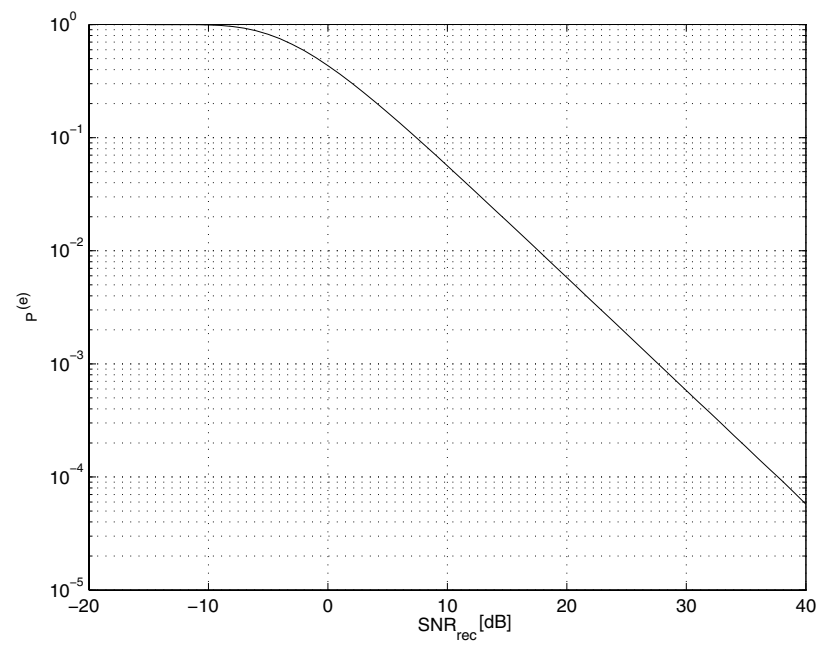

Fig. 6. Frame error probability $P^{(e)}$ versus SNR at the detector stage of the receiver $\left(S N R_{r e c}\right)$. The RCPC code used has rate $1 / 2$, parent code rate of $1 / 4$, puncturing period of 8 , and memory of 4 . The receiver noise figure $(F)$ is $5 \mathrm{~dB}$.

with [22], $\gamma=300$. Traffic is uniform, i.e., $\lambda_{i}=1, \forall i \in \mathcal{N}$. The formulations presented in Section III are solved using LP Solve 5.5.0.7 [23].

The transmission energy per bit $E_{b}$, i.e., the value of energy that is radiated by a sensor node to transmit one bit, is assumed to be the same at each sensor node. Unless otherwise specified, the energy consumed to receive and process one bit at the sensor node receiver is $E_{b}^{(R x)}=30 \mathrm{~nJ}$ [24].

Figs. 7 and 8 show the value of (15) for a simple network with one base-station $B S$ and 2 sensor nodes $s$ and $r$. The value of upper bound $\Psi_{(s, r)}$ on the saturation throughput is plotted as a function of the coordinates of $r$. Coordinates of $s$ and $B S$ are $(50,0)$ and $(0,0)$, respectively. Fig. 8 shows the $y=0 \mathrm{~m}$ section of the three dimensional plot of Fig. 7. The following parameter values are used: $\lambda_{s}=\lambda_{r}=1$ packet/s, $E_{b_{s}}=E_{b_{r}}=2 e^{-12} \mathrm{~J}, E_{b}^{(R x)}=0 \mathrm{~J}$, and $\gamma=1$. Not surprisingly, both Figs. 7 and 8 indicate that cooperation is most effective when $r$ is placed between $s$ and $B S$.

Additionally, Fig. 8, reports the value of $S_{A R Q-N C}$ expressed in (16). For the set of parameter values chosen in this example, cooperation may increase the saturation throughput up to three times.

As the value of the saturation throughput depends heavily on the sensor node positions, multiple simulation experiments are performed. Each experiment is obtained by randomly placing 300 sensor nodes within a circular footprint of radius $R$ according to a uniform distribution. The base-station is at the footprint center. The number of simulation experiments is chosen such that the average value for the saturation throughput $S$ has a confidence interval of $8 \%$ or better at 98\% confidence level.

Fig. 9 plots the saturation throughput $S$ versus the transmission energy per bit $E_{b} . A R Q-C(300)$ refers to the case of cooperative ARQ protocols with no constraints on the number of relays per source. $A R Q-C(1)$ refers to the one-relay cooperative ARQ protocols. All curves show a

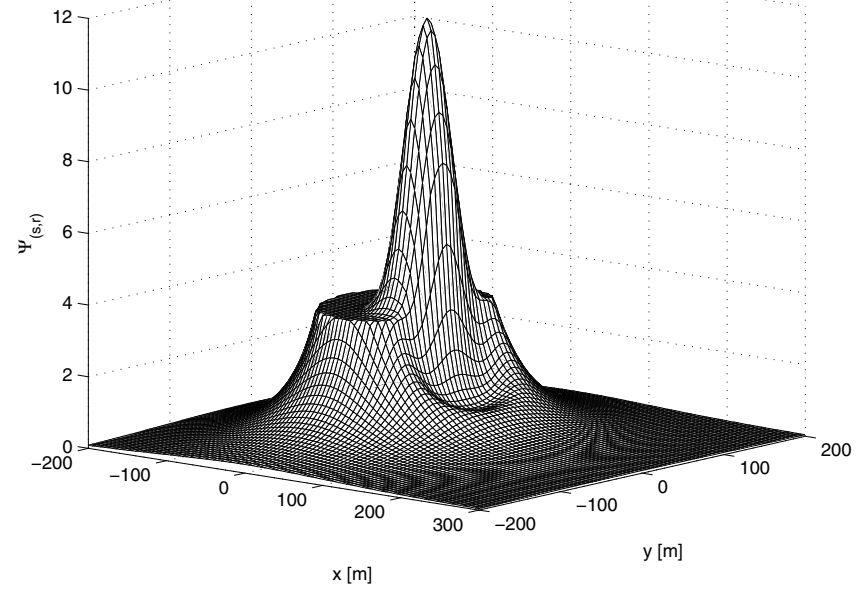

Fig. 7. Upper bound on the saturation throughput $\left(\Psi_{(s, r)}\right)$ as a function of the position of sensor node $r$. Sensor node $s$ is placed in $(50,0)$ and the base-station is placed in $(0,0)$.

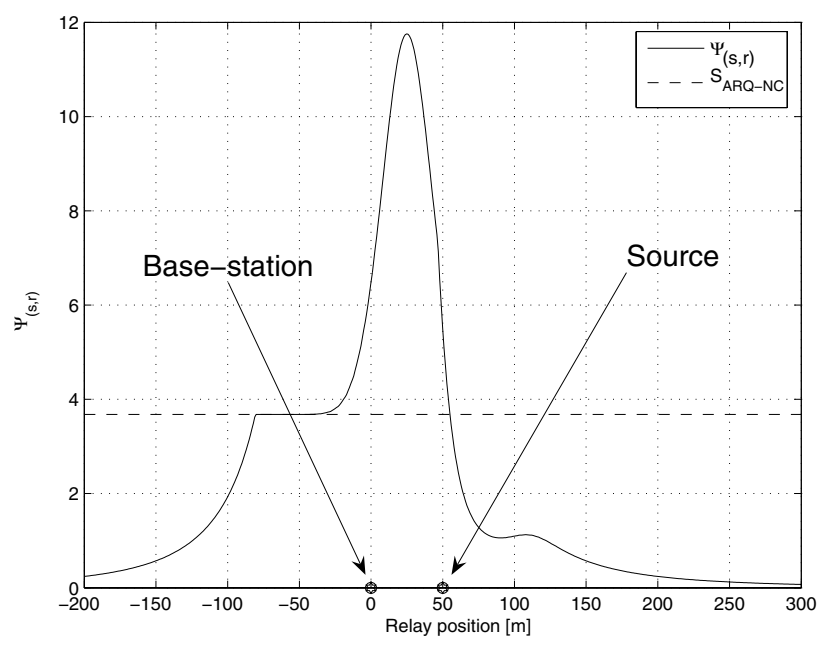

Fig. 8. Upper bound on the saturation throughput $\left(\Psi_{(s, r)}\right)$ as a function of the position of sensor node $r$. Sensor node $s$ is placed in $(50,0)$, the base-station is placed in $(0,0)$ and the $y$ coordinate of the relay $r$ is fixed to $0 \mathrm{~m}$.

similar pattern, with a maximum at some intermediate value of $E_{b}$. Higher values of $E_{b}$ increase both the SNR and the probability of successful transmission, but consume too much energy. In this case, all ARQ protocols perform similarly, as retransmission attempts are sporadic. Lower values of $E_{b}$ save energy on the single transmission attempt, but require too many retransmission attempts. In this case, the two classes of ARQ protocols behave in a significantly different manner from one another. The ARQ-C protocols achieve their maximum $S$ at a $E_{b}$ value that is slightly lower than the value of the ARQ-NC protocols. This fact is explained by the combined effect of energy borrowing and relay retransmission of the ARQ-C protocols. Sensor nodes whose energy consumptionto-recharge rate ratio is not favorable, can borrow energy from other (energy richer) sensor nodes by asking them to 


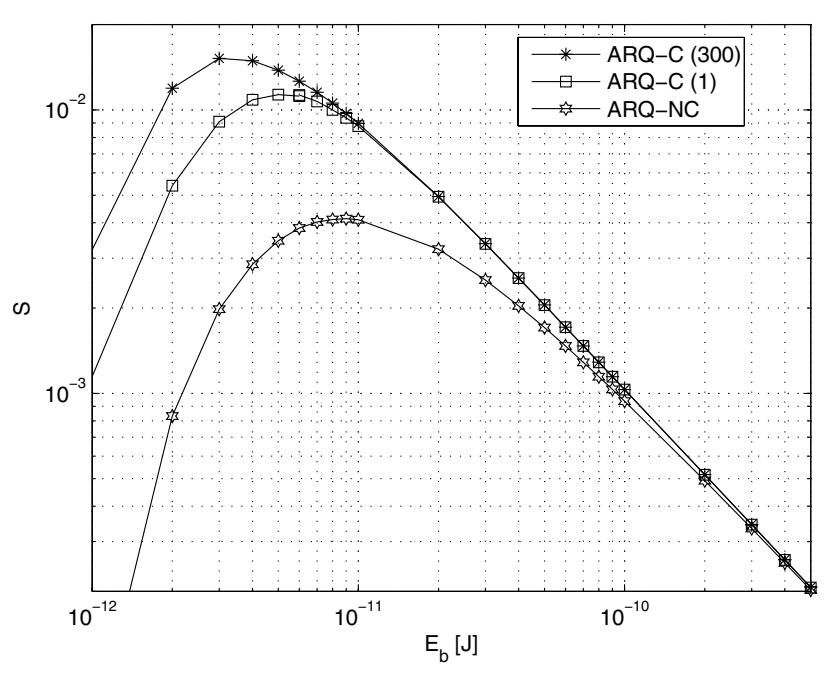

Fig. 9. Saturation throughput $(S)$ versus energy per bit transmitted at the sensor nodes $\left(E_{b}\right) . R=50 \mathrm{~m}, G=1, \gamma=300, F=5 \mathrm{~dB}, E_{b}^{(R x)}=30 \mathrm{~nJ}$, $n=3$.

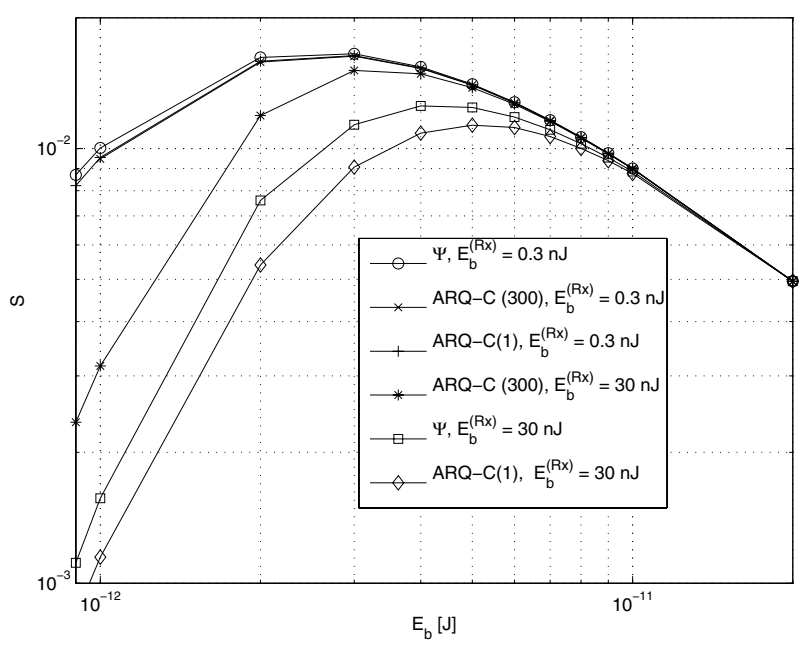

Fig. 10. Saturation throughput $(S)$ versus energy per bit transmitted at the sensor nodes $\left(E_{b}\right) . R=50 \mathrm{~m}, G=1, \gamma=300, F=5 \mathrm{~dB}, n=3$.

perform data frame retransmissions. At the same time, the transmission energy level at the sensor node may be lowered without compromising throughput due to the shorter transmission range offered by the relay retransmission mechanism. Note that lowering the transmitted energy level has the initial effect of increasing the amount of retransmissions performed by the relay, i.e., more energy is borrowed from the relay. Further lowering of the transmitted energy level compromises the ability of the relay to help, causing a sharp throughput decay. Finally, the plots indicate that, when the energy amount available at the sensor node is the limiting factor in the system, the saturation throughput achieved by the ARQ-C protocols, may be more than twice the saturation throughput of the ARQ$\mathrm{NC}$ protocols. In addition, limiting to number of relays per source to one seems to be a good trade-off between network throughput and protocol complexity.

Fig. 10 shows the effect of $E_{b}^{(R x)}$ on $S$. Two values are considered, $E_{b}^{(R x)}=30 \mathrm{~nJ}$ and $E_{b}^{(R x)}=0.3 \mathrm{~nJ}$. As intuition suggests, $S$ grows with decreasing $E_{b}^{(R x)}$. The figures also shows the upper bound for $A R Q-C(1)$ derived in Section IVB. Note that the performance gap between $A R Q-C(300)$ and $A R Q-C(1)$ decreases with decreasing $E_{b}^{(R x)}$.

Table II documents the effect of the uplink frequency $\left(f_{u p}\right)$, the footprint radius $(R)$, and the path loss coefficient $(n)$ on $S$ for the two classes of ARQ protocols. Results are compared against an upper bound of the multi-hop MEP protocol throughput. The upper bound of the MEP protocol throughput is computed assuming that $L_{A C K}=0$ bits, i.e., transmitting and receiving ACK frames do not consume energy at the sensor nodes. Two values of $E_{b}^{(R x)}$ are considered, i.e., $E_{b}^{(R x)}=30 \mathrm{~nJ}$ and $E_{b}^{(R x)}=0.3 \mathrm{~nJ}$. The $S$ values reported in the table are obtained using the value for $E_{b}$ that yields the maximum $S$ value for each solution. Results in the top part of the table are obtained using an uplink frequency $f_{u p}=$ $2.4 \mathrm{GHz}$. Results in the middle part of the table are obtained using an uplink frequency $f_{u p}=916.5 \mathrm{MHz}$. Results in bottom part of the table are obtained using an uplink frequency $f_{u p}=433.92 \mathrm{MHz}$. Depending on the surrounding environment - which may affect the value of $n$ - the practical size of the footprint spans from tens to hundred meters. Even though the ARQ-C protocols always yields higher saturation throughput values than the ones achieved by the ARQ-NC protocols, noticeable performance differences are found only in those cases when the direct transmission of data frames from the sensor node to the base-station is not favored by the surrounding environment, e.g., for increasing values of $f_{u p}$, $R$ and $n$. Similar results are found under non uniform traffic patterns, which are discussed in [10]. For even larger values of $f_{u p}, R$ and $n$ the multi-hop MEP protocol is the best solution. This is the case when the relay based approach used in the $A R Q-C$ protocols might not suffice to compensate for the noisy uplink channel from the source to the base-station.

\section{CONCLUSION}

To ensure reliable sensor node data collection in energy harvesting wireless sensor networks, two classes of ARQ protocols - one of which is based on cooperative communications - were considered and compared. It was found that when the energy available at the sensor node is the main limiting factor in the system, the cooperative ARQ saturation throughput may be more than twice the saturation throughput of the non-cooperative ARQ protocols. Cooperating sensor nodes are able to effectively balance their energy consumption-torecharge rate ratio by borrowing energy from one another during the data frame retransmission phase. At the same time, the transmission energy level at the sensor node may be lowered without compromising throughput due to the shorter transmission range offered by the relay retransmission mechanism. It was also found that limiting the number of relays per source to one constitutes a good trade-off between network performance and protocol complexity.

\section{ACKNOWLEDGMENT}

The authors would like to thank Isabella Cerutti, Stefano Gregori, Franco Maloberti, and Aria Nosratinia for their valuable technical conversations and input. 
TABLE II

SATURATION THROUGHPUt $(S)$ AS A FUNCTION OF THE UPLINK FREQUENCY $\left(f_{u p}\right)$, FOOTPRINT RADIUS $(R)$, AND PATH LOSS COEFFICIENT $(n)$

\begin{tabular}{|c|c|c|c|c|c|c|c|c|c|c|}
\hline \multicolumn{11}{|c|}{$f_{u p}=2.4 \mathrm{GHz}, G_{T}=G_{R}=1, \gamma=300, F=5 \mathrm{~dB}$} \\
\hline & \multicolumn{5}{|c|}{$E_{b}^{(R x)}=30 \mathrm{~nJ}$} & \multicolumn{5}{|c|}{$E_{b}^{(R x)}=0.3 \mathrm{~nJ}$} \\
\hline & \multicolumn{3}{|c|}{$n=3$} & \multicolumn{2}{|c|}{$R=50 \mathrm{~m}$} & \multicolumn{3}{|c|}{$n=3$} & \multicolumn{2}{|c|}{$R=50 \mathrm{~m}$} \\
\hline & $R=10 \mathrm{~m}$ & $R=50 \mathrm{~m}$ & $R=100 \mathrm{~m}$ & $n=2$ & $n=4$ & $R=10 \mathrm{~m}$ & $R=50 \mathrm{~m}$ & $R=100 \mathrm{~m}$ & $n=2$ & $n=4$ \\
\hline ARQ-NC & 64.3 & $4.1 \mathrm{E}-3$ & $6.4 \mathrm{E}-5$ & 10.2 & $1.6 \mathrm{E}-6$ & 64.3 & $4.1 \mathrm{E}-3$ & $6.4 \mathrm{E}-5$ & 10.2 & $1.6 \mathrm{E}-6$ \\
\hline ARQ-C(1) & 112.4 & $1.1 \mathrm{E}-2$ & $2.4 \mathrm{E}-4$ & 10.9 & $1.2 \mathrm{E}-5$ & 170.9 & $1.6 \mathrm{E}-2$ & $2.6 \mathrm{E}-4$ & 20.5 & $1.2 \mathrm{E}-5$ \\
\hline ARQ-C(300) & 124.1 & $1.5 \mathrm{E}-2$ & $2.5 \mathrm{E}-4$ & 13.9 & $1.2 \mathrm{E}-5$ & 232.2 & $1.6 \mathrm{E}-2$ & $2.6 \mathrm{E}-4$ & 24.9 & $1.2 \mathrm{E}-5$ \\
\hline MEP & 65.1 & $4.9 \mathrm{E}-3$ & $1.4 \mathrm{E}-4$ & 10.3 & $1.8 \mathrm{E}-5$ & 71.6 & $2.7 \mathrm{E}-2$ & $1.3 \mathrm{E}-3$ & 8.3 & $2.6 \mathrm{E}-4$ \\
\hline \multicolumn{11}{|c|}{$f_{u p}=916.5 \mathrm{MHz}, G_{T}=G_{R}=1, \gamma=300, F=5 \mathrm{~dB}$} \\
\hline & \multicolumn{5}{|c|}{$E_{b}^{(R x)}=30 \mathrm{~nJ}$} & \multicolumn{5}{|c|}{$E_{b}^{(R x)}=0.3 \mathrm{~nJ}$} \\
\hline & \multicolumn{3}{|c|}{$n=3$} & \multicolumn{2}{|c|}{$R=50 \mathrm{~m}$} & \multicolumn{3}{|c|}{$n=3$} & \multicolumn{2}{|c|}{$R=50 \mathrm{~m}$} \\
\hline & $R=10 \mathrm{~m}$ & $R=50 \mathrm{~m}$ & $R=100 \mathrm{~m}$ & $n=2$ & $n=4$ & $R=10 \mathrm{~m}$ & $R=50 \mathrm{~m}$ & $R=100 \mathrm{~m}$ & $n=2$ & $n=4$ \\
\hline ARQ-NC & 440.7 & $2.8 \mathrm{E}-2$ & $4.4 \mathrm{E}-4$ & 69.7 & $1.1 \mathrm{E}-5$ & 440.7 & $2.8 \mathrm{E}-2$ & $4.4 \mathrm{E}-4$ & 69.7 & $1.1 \mathrm{E}-5$ \\
\hline ARQ-C(1) & 597.2 & $5.6 \mathrm{E}-2$ & $1.2 \mathrm{E}-3$ & 70.5 & 7.5E-5 & 843.9 & $11.2 \mathrm{E}-2$ & $1.8 \mathrm{E}-3$ & 122.3 & $8.2 \mathrm{E}-5$ \\
\hline ARQ-C(300) & 731.5 & 7.6E-2 & $1.6 \mathrm{E}-3$ & 78.9 & $8.1 \mathrm{E}-5$ & $1.1 \mathrm{E} 3$ & $11.2 \mathrm{E}-2$ & $1.8 \mathrm{E}-3$ & 149.8 & $8.2 \mathrm{E}-5$ \\
\hline MEP & 444.5 & $2.4 \mathrm{E}-2$ & $5.4 \mathrm{E}-4$ & 70.1 & $5.3 \mathrm{E}-5$ & 369.9 & 7.7E-2 & $3.2 \mathrm{E}-3$ & 70.3 & $5.9 \mathrm{E}-4$ \\
\hline \multicolumn{11}{|c|}{$f_{u p}=433.92 \mathrm{GHz}, G_{T}=G_{R}=1, \gamma=300, F=5 \mathrm{~dB}$} \\
\hline & \multicolumn{5}{|c|}{$E_{b}^{(R x)}=30 \mathrm{~nJ}$} & \multicolumn{5}{|c|}{$E_{b}^{(R x)}=0.3 \mathrm{~nJ}$} \\
\hline & \multicolumn{3}{|c|}{$n=3$} & \multicolumn{2}{|c|}{$R=50 \mathrm{~m}$} & \multicolumn{3}{|c|}{$n=3$} & \multicolumn{2}{|c|}{$R=50 \mathrm{~m}$} \\
\hline & $R=10 \mathrm{~m}$ & $R=50 \mathrm{~m}$ & $R=100 \mathrm{~m}$ & $n=2$ & $n=4$ & $R=10 \mathrm{~m}$ & $R=50 \mathrm{~m}$ & $R=100 \mathrm{~m}$ & $n=2$ & $n=4$ \\
\hline ARQ-NC & $1.9 \mathrm{E} 3$ & $12.6 \mathrm{E}-2$ & $2.0 \mathrm{E}-3$ & 314.9 & $4.8 \mathrm{E}-5$ & $1.9 \mathrm{E} 3$ & $12.6 \mathrm{E}-2$ & $2.0 \mathrm{E}-3$ & 314.9 & $4.8 \mathrm{E}-5$ \\
\hline ARQ-C(1) & $2.1 \mathrm{E} 3$ & $22.4 \mathrm{E}-2$ & $4.2 \mathrm{E}-3$ & 315.5 & $2.5 \mathrm{E}-4$ & $3.5 \mathrm{E} 3$ & $42.4 \mathrm{E}-2$ & $7.8 \mathrm{E}-3$ & 406.3 & $3.6 \mathrm{E}-4$ \\
\hline ARQ-C(300) & $2.6 \mathrm{E} 3$ & $27.7 \mathrm{E}-2$ & $5.6 \mathrm{E}-3$ & 335.1 & $3.3 \mathrm{E}-4$ & $4.1 \mathrm{E} 3$ & $49.2 \mathrm{E}-2$ & $7.8 \mathrm{E}-3$ & 530.8 & 3.6E-4 \\
\hline MEP & $1.9 \mathrm{E} 3$ & $11.9 \mathrm{E}-2$ & $1.8 \mathrm{E}-3$ & 316.5 & $1.3 \mathrm{E}-4$ & $1.9 \mathrm{E} 3$ & $21.2 \mathrm{E}-2$ & $6.7 \mathrm{E}-3$ & 316.5 & $1.0 \mathrm{E}-3$ \\
\hline
\end{tabular}

\section{REFERENCES}

[1] I. Akyildiz, W. Su, Y. Sankarasubramaniam, and E. Cayirci, "Wireless sensor networks: a survey," Elsevier Computer Networks, vol. 38, no. 4, pp. 393-442, 2002.

[2] W. R. Heinzelman, A. Chandrakasan, and H. Balakrishnan, "Energy efficient protocol for wireless microsensor networks," in Proc. Hawaii International Conference on System Sciences (HICSS), Jan. 2000, pp. $1-10$.

[3] G. Asada, M. Dong, T. Lin, F. Newberg, G. Pottie, W. Kaiser, and H. Marcy, "Wireless integrated network sensors: low power systems on a chip," in Proc. 1998 European Solid State Circuits Conference, Oct. 1998.

[4] J. M. Rabaey, M. J. Ammer, J. L. da Silva Jr., D. Patel, and S. Roundy, "PicoRadio supports ad hoc ultra-low power wireless networking," IEEE Computer, vol. 33, no. 7, pp. 42-48, 2000.

[5] B. H. Calhoun, D. C. Daly, N. Verma, D. F. Finchelstein, D. D Wentzloff, A. Wang, S.-H. Cho, and A. P. Chandrakasan, "Design considerations for ultra-low energy wireless microsensor nodes," IEEE Trans. Comput., vol. 54, no. 6, pp. 727-740, 2005.

[6] "SensoNet Project," http://users.ece.gatech.edu/ weilian/Sensor.

[7] "WSSN (Wireless Self-Sustaing Sensor Network) Project," http://www.ict.tuwien.ac.at/wireless/.

[8] S. Roundy, P. K. Wright, and J. M. Rabaey, Energy Scavenging for Wireless Sensor Networks: With Special Focus on Vibrations. Norwell, MA: Kluwer Academic, 2004.

[9] S. Mahlknecht, "Energy-self-sufficient wireless sensor networks for the home and building environment," Ph.D. dissertation, Vienna University of Technology, Vienna, Austria, Sept. 2004. [Online]. Available: http://www.ict.tuwien.ac.at/wireless/pubblications.html

[10] P. Monti, "Achieving reliable networking for the generic autonomous platform for sensor systems (GAP4S)," Ph.D. dissertation, Univ. of Texas at Dallas, Richardson, TX, USA, Dec. 2005.

[11] D. Bertsekas and R. Gallager, Data Networks (Second Edition). Upper Saddle River, NJ: Prentice Hall, 1992.
[12] T. Cover and A. Gamal, "Capacity theorems for the relay channel," IEEE Trans. Inf. Theory, vol. 25, no. 5, pp. 572-584, 1979.

[13] A. Sendonaris, E. Erkip, and B. Aazhang, "User cooperation diversitypart I: system description," IEEE Trans. Commun., vol. 51, no. 11, pp. 1927-1938, 2003.

[14] M. Janani, A. Hedyat, T. Hunter, and A. Nosratinia, "coded cooperation in wireless communications: space-time transmission and iterative decoding," IEEE Trans. Signal Processing, vol. 52, no. 2, pp. 362-371, 2004

[15] J. N. Laneman, D. N. C. Tse, and G. W. Wornell, "Cooperative diversity in wireless networks: efficient protocols and outage behavior," IEEE Trans. Inf. Theory, vol. 50, no. 12, pp. 3062-3080, 2004.

[16] B. Zhao and M. C. Valenti, "Practical relay networks: a generalization of hybrid-ARQ," IEEE J. Sel. Areas Commun., vol. 23, no. 1, pp. 7-18, 2005.

[17] E. Zimmermann, P. Herhold, and G. Fettweis, "On the performance of cooperative relaying protocols in wireless networks," European Trans. Telecommun. (ETT), vol. 16, no. 1, pp. 17-35, 2005.

[18] I. Cerutti, A. Fumagalli, and P. Gupta, "Delay models of single-source single-relay cooperative ARQ protocols in slotted radio networks with Poisson frame arrivals," IEEE/ACM Trans. Networking, to appear.

[19] T. H. Cormen, C. E. Leiserson, R. L. Rivest, and C. Stein, Introduction to Algorithms (Second Edition). Cambridge, MA: MIT Press, 2001.

[20] H. Karloff, Linear Programming. Boston, MA: Birkhäuser, 2001.

[21] J. Hagenauer, "Rate-compatible punctured convolutional codes (RCPC codes) and their applications," IEEE Trans. Commun., vol. 36, no. 4, pp. 389-400, 1988.

[22] Y. Sankarasubramaniam, I. F. Akyildiz, and S. W. McLaughlin, "Energy efficiency based packet size optimization in wireless sensor networks," in Proc. IEEE First International Workshop on Sensor Network Protocols and Applications, May 2003, pp. 1-8.

[23] "Lp solve 5.5.5.10," http://lpsolve.sourceforge.net/5.5/.

[24] "Datasheet CC2400 (Rev 1.5)," http://www.chipcon.com. 


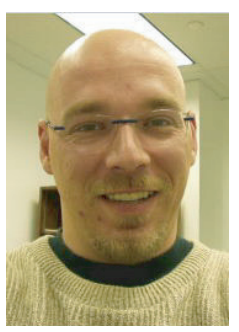

Marco Tacca joined the University of Texas at Dallas in August 2002 as a senior lecturer. Marco Tacca received his Laurea Degree from Politecnico di Torino in 1998 and $\mathrm{PhD}$ from the University of Texas at Dallas in 2002. Marco Tacca's research interests include aspects of optical networks, high speed photonic network planning, fault protection and restoration with particular emphasis on aspect of differentiated reliability, as well as sensor and wireless networks.

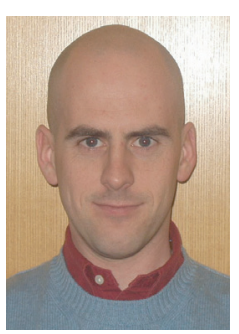

Paolo Monti received a Laurea degree in Electrical Engineering (2001) from the Politecnico di Torino, Italy, and a Ph.D. in Electrical Engineering (2005) from the University of Texas at Dallas (UTD). Since January 2005 he is a Post-Doctoral Research Associate of the Open Networking Advance Research (OpNeAR) Lab at UTD. His research interests include network planning, protocol design, performance evaluation and optimization techniques for both optical and sensor/ad-hoc networks. He is a member of IEEE ComSoc.

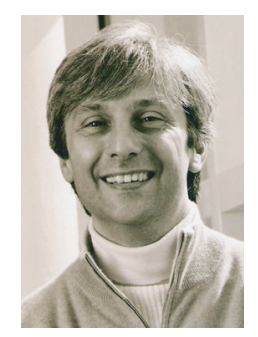

Andrea Fumagalli received his Ph.D. from Politecnico di Torino, Italy, and is a Professor of Electrical Engineering at the University of Texas at Dallas. Prior to joining UTD he was an Assistant Professor of the Electronics Engineering Department at the Politecnico di Torino, Italy. He is a member of the Open Networking Advanced Research (OpNeAR) Lab at UTD (http://opnear.utdallas.edu).

His research interests include aspects of wired and wireless networks, and related protocol design and performance evaluation. He has been involved in a number of research projects focusing on packet switched and faulttolerant networks, and has published more than one hundred papers in peer reviewed journals and conferences. He serves on the Editorial Board of the ACM/IEEE Transactions on Networking and two Elsevier journals. He was on a Distinguished Lecturer Tour for IEEE ComSoc in 2000. 